\title{
Efeito da caminhada em esteira na acuidade proprioceptiva do tornozelo em mulheres jovens saudáveis: ensaio clínico randomizado
}

\author{
Effect of treadmill walking on ankle proprioceptive acuity in healthy young women: \\ a randomized clinical trial
}

Ronaldo Valdir Briani ${ }^{1} \bowtie$, Carolina Silva Flóride ${ }^{2}$, Fernanda Elisa Ribeiro ${ }^{1}$, Giovanna Altero Arévalo ${ }^{1}$, Guilherme Yassuyuki Tacao ${ }^{1}$, Luana Almeida Gonzaga', Maíra Caroline de Oliveira ${ }^{3}$, Ana Paula Borges Brasil ${ }^{4}$, Diego Giulliano Destro Christofaro', José Mohamud Vilagra²

\author{
1 Programa de Pós-Graduação Stricto Sensu em Fisioterapia, Faculdade de Ciências e Tecnologia, Universidade Estadual Paulista Júlio de Mesquita Filho (UNESP). \\ Presidente Prudente, SP. \\ 2 Universidade Estadual do Oeste do Paraná (UNIOESTE). Cascavel, PR. \\ 3 Programa de Pós-Graduação em Fisioterapia. Universidade do Estado de Santa Catarina (UESC). Florianópolis, SC. \\ ${ }^{4}$ Programa de Residência de Fisioterapia em Pediatria, Universidade Estadual de Londrina (UEL). Londrina, PR.
}

\section{RESUMO}

Objetivos: Avaliar o efeito da caminhada em esteira na acuidade proprioceptiva do tornozelo de mulheres jovens saudáveis.

Métodos: Para este ensaio clínico randomizado foram selecionadas 20 mulheres entre 18 e 30 anos com um nível baixo de atividade física segundo a classificação IPAQ (Questionário Internacional de Nível de Atividade Física). As participantes foram divididas em dois grupos de 10 componentes, o Grupo Exercício, que realizou 10 sessões de caminhada em esteira, e o Grupo Controle, que não foi submetido a nenhuma intervenção. A acuidade proprioceptiva do tornozelo foi avaliada pré e pós-intervenção por meio do Star Excursion Balance Test. Após ser confirmada a normalidade dos dados, foi utilizada a análise de variância com delineamento misto para comparação entre os grupos e entre as avaliações pré e pós-intervenção.

Resultados: Não foram encontradas diferenças significativas na execução do Star Excursion Balance Test comparando as avaliações pré e pós-intervanção em ambos os grupos. Também não foram encontradas diferenças entre os dois grupos comparando as variações percentuais entre as duas avaliações.

Conclusões: Nas condições testadas, um programa de 10 sessões de caminhada em esteira não foi capaz de promover adaptações na acuidade proprioceptiva do tornozelo em mulheres jovens saudáveis.

DESCRITORES: propriocepção; caminhada; equilíbrio postural.

\section{ABSTRACT}

Aims: To evaluate the effect of treadmill walking on ankle proprioceptive acuity in healthy young women.

Methods: This randomized clinical trial included 20 women aged 18 to 30 years with a low level of physical activity according to the International Physical Activity Questionnaire classification. The participants were assigned to two groups with 10 components: the exercise group, which performed 10 treadmill walking sessions, and the control group, which was not submitted to any kind of intervention. Ankle proprioceptive acuity was assessed before and after the Star Excursion Balance Test. After confirming the normality of data, a mixed analysis of variance was used to compare the groups and the assessments before and after the intervention.

Results: There were no significant differences in the Star Excursion Balance Test between groups either before or after the intervention. In addition, intergroup differences were not found when percentage variations were compared in both groups.

Conclusions: Under the tested conditions, 10 treadmill walking sessions were not able to produce proprioceptive adaptations in healthy young women.

KEY WORDS: proprioception; walking; postural balance. 
Abreviaturas: SNC, sistema nervoso central; IPAQ, questionário internacional de atividade física; SEBT, star excursion balance test; ANOVA, análise de variância; GE, grupo exercício; GC, grupo controle; LAPEMH, Laboratório de Pesquisa de Equilíbrio e Movimento Humano; UNIOESTE, Universidade Estadual do Oeste do Paraná.

\section{INTRODUÇÃO}

A propriocepção é um subcomponente restrito à via aferente do sistema sensório-motor, que engloba todas as informações mecânicas originadas pelos mecanorreceptores musculares e tendíneos, além de receptores localizados nos ligamentos, cápsula articular, meniscos e tecidos cutâneos [1]. Estes transduzem os eventos mecânicos que ocorrem nos tecidos corporais em sinais neurais, resultando em informações sensoriais transmitidas ao sistema nervoso central (SNC) [2]. O SNC, por sua vez, após processar centralmente tais informações, gera respostas motoras a um estímulo aferente, tanto inconscientes quanto conscientes, as quais contribuem para a estabilidade articular dinâmica [3].

Dessa forma, o sistema proprioceptivo é de grande importância, pois fornece sinais que servirão de base para o planejamento e controle motor e contribuirão para a regulação da estabilidade articular durante o movimento [4]. A realização adequada do movimento está intimamente interligada à acuidade do sistema proprioceptivo. A ocorrência de lesões em atividades físicas cotidianas e práticas esportivas está relacionada ao funcionamento alterado desse sistema, tanto como causa quanto como consequência [1].

Dentre as articulações do corpo humano, a articulação do tornozelo é uma das mais importantes para o controle do equilíbrio corporal durante o esporte e atividades cotidianas. A propriocepção do tornozelo fornece informações essenciais para os ajustes corporais de quase todas as outras articulações devido ao seu contato precoce com o solo. Dessa forma, a atividade proprioceptiva adequada dessa articulação é de grande importância não apenas para o seu funcionamento, mas também para a função de diversas articulações do corpo humano. Perturbações na funcionalidade da propriocepção de tornozelo vêm sendo relacionadas com a ocorrência de diversas lesões ortopédicas e alterações do controle postural, tanto na prática esportiva quanto em atividades de vida diária [1,3-5].

Há uma crescente demanda em clínicas ortopédicas e hospitais por causa de lesões ortopédicas, principalmente lesões articulares e ligamentares de diversas articulações, mais frequentemente do tornozelo [6]. Especificamente as mulheres, tanto atletas como não atletas, são mais susceptíveis a lesões articulares de joelho e tornozelo por causa de alterações proprioceptivas, juntamente com demais fatores musculoesqueléticos [4,7]. Tal demanda gera notável gasto orçamentário que poderia ser reduzido por condutas preventivas focadas no reestabelecimento ou aprimoramento proprioceptivo [8]. No entanto, exercícios que visam a aprimorar a acuidade proprioceptiva demandam aparelhos terapêuticos específicos e supervisão profissional capacitada para sua aplicação [1]. Assim, grande parte da população não se beneficia dessa abordagem em razão de dificuldades econômicas e/ou falta de contingente profissional capacitado para a aplicação de tais exercícios [9].

Alternativamente, a prática regular de atividade física, como a caminhada e/ou corrida, vem crescendo como forma abrangente de tratamento e prevenção de doenças [10]. Estudos recentes sugerem a caminhada como adjuvante para o controle ponderal, regulação hormonal e diminuição dos riscos cardiovasculares $[10,11]$. No entanto, pouco se tem pesquisado sobre o efeito da caminhada sobre a propriocepção articular. De fato, ao conhecimento dos autores, não foram encontrados estudos na literatura que tivessem avaliado tal efeito. Informações referentes a este assunto podem elucidar um possível fator benéfico da caminhada no contexto proprioceptivo. Ou seja, é possível que a prática regular de atividade física, como a caminhada, possa promover ganhos proprioceptivos e, por conseguinte, influenciar positivamente a prevenção de lesões musculoesqueléticas.

Dessa forma, o objetivo desse estudo foi avaliar se um programa de atividade física regular, como a caminhada em esteira, seria capaz de promover aumento da acuidade proprioceptiva do tornozelo de mulheres jovens saudáveis.

\section{MÉTODOS}

\section{Amostra e local da pesquisa}

A amostra foi composta por mulheres na faixa etária entre 18 e 30 anos, convidadas a participar do estudo via anúncios alocados na Universidade Estadual do Oeste do Paraná (UNIOESTE). Baseado em um estudo prévio [12], foi realizado o cálculo amostral para encontrar uma diferença entre avaliações de 2,8 $\mathrm{cm}( \pm 5,8 \mathrm{~cm}$ de desvio padrão) com um alfa de 0,05 . Com base nos cálculos realizados no software SPSS, sete indivíduos por grupo são necessários para obter $80 \%$ de poder estatístico. 
Para serem incluídas no estudo as voluntárias deveriam ser classificadas com baixo nível de atividade física por meio do Questionário Internacional de Atividade Física (IPAQ) versão longa, ferramenta esta validada e reprodutível para este fim $[13,14]$ não participar de outro treinamento; e não relatar histórico de lesões ortopédicas. Foram selecionadas mulheres com baixo nível de atividade física para impedir que as participantes já tivessem contato regular com a intervenção proposta. Ou seja, foram incluídas apenas mulheres que não realizavam a prática regular da caminhada, ou qualquer outra atividade física mais que duas vezes por semana.

Os critérios de exclusão foram lesões musculoesqueléticas nos últimos seis meses; lesões musculares crônicas incapacitantes; lesões neurológicas ou utilização de drogas que afetam o equilíbrio; afecções cardiovasculares; histórico de cirurgia no aparelho locomotor; diabetes; utilizar sedativos ou ansiolíticos; lesionar-se e necessitar de tratamento médico e/ou fisioterapêutico durante a pesquisa; fazer uso de bebida alcoólica ou realizar alguma atividade que envolva exercícios intensos nas 12 horas que antecederam os testes; faltar a três sessões consecutivas ou a quatro sessões não consecutivas; e ausentar-se nos períodos das avaliações.

As participantes foram divididas aleatoriamente em dois grupos. A atribuição dos grupos foi realizada usando envelopes opacos e lacrados, cada um contendo o nome de um dos grupos. Os envelopes foram escolhidos por um indivíduo não envolvido com a pesquisa. O Grupo Exercício (GE) foi submetido a sessões de caminhada em esteira ergométrica e o Grupo Controle (GC) não participou do protocolo de treinamento e não teve suas atividades diárias afetadas.

O projeto de pesquisa foi aprovado pelo Comitê de Ética em Pesquisa da Universidade Estadual do Oeste do Paraná (parecer no 393.674/2013). Antes de serem iniciadas as coletas de dados, todas as participantes concordaram em assinar o Termo de Consentimento Livre e Esclarecido. $O$ estudo foi realizado no Laboratório de Pesquisa de Equilíbrio e Movimento Humano (LAPEMH) da UNIOESTE, onde foram executadas todas as avaliações.

\section{Avaliações pré-intervenção e pós-intervenção}

As avaliações pré-intervenção ocorreram em um único dia, e as avaliações pós-intervenção aconteceram ao término do protocolo de treinamento, sendo idênticas às avaliações iniciais pré-intervenção.
Previamente a cada avaliação foram mensurados os dados de massa corporal e altura de cada participante com balança digital (Relaxmedic ${ }^{\circledR}$, São Paulo, SP) e fita métrica. Para avaliação da acuidade proprioceptiva do tornozelo foi utilizado o instrumento Star Excursion Balance Test (SEBT), ferramenta validada e reprodutível [3]. Para a sua execução, a participante deve se manter na posição unipodal com seu membro dominante, enquanto o membro contralateral alcança a maior distância possível ao longo da linha escolhida e retorna para a posição inicial enquanto mantém o equilíbrio (Figura 1). O examinador, que era cego quanto aos grupos, marcava o ponto mais distante tocado ao longo da linha com um lápis e, posteriormente, media a distância em centímetros, do centro da grade até o ponto de toque do pé. Foi realizada uma tentativa para cada participante. A distância alcançada no teste era mensurada por meio de fita métrica. Caso o pé de apoio fosse retirado do local, o teste era interrompido e repetido.

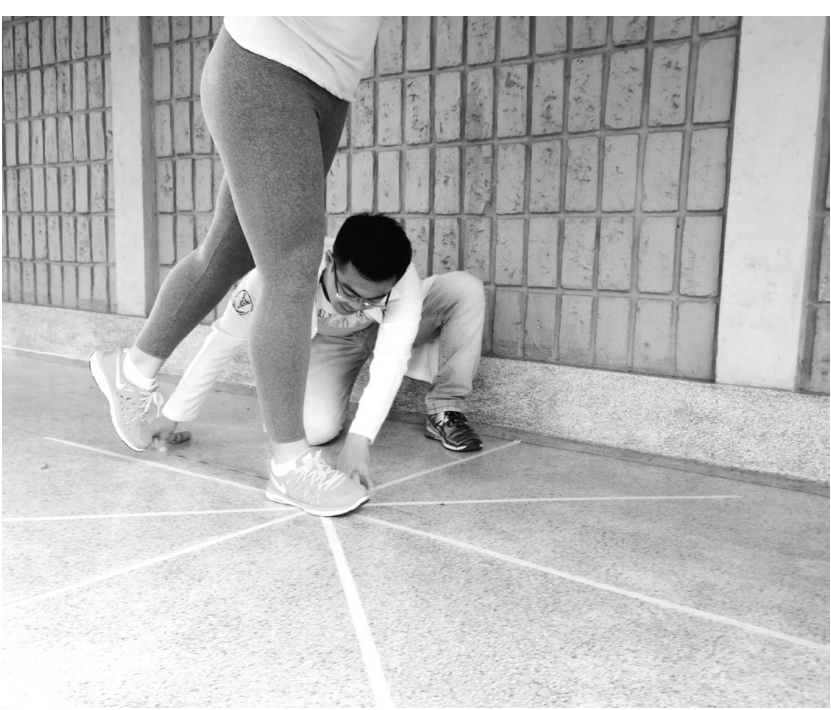

Figura 1. Posicionamento para execução do Star Excursion Balance Test (SEBT) para medida da acuidade proprioceptiva do tornozelo.

O tornozelo avaliado era o do membro que se mantinha fixo no solo. As linhas de direção eram: M1 - distância entre o ponto central e a linha anterior; M2 - distância entre o ponto central e a linha ânteromedial; M3 - distância entre o ponto central e a linha medial; M4 - distância entre o ponto central e a linha póstero-medial; M5 - distância entre o ponto central e a linha posterior; L1 - distância entre o ponto central e a linha anterolateral; L2 - distância entre o ponto 
central e a linha lateral; L3 - distância entre o ponto central e a linha póstero-lateral. O teste foi executado com cada participante utilizando seu próprio calçado, sendo que o mesmo calçado foi utilizado durante a intervenção em esteira ergométrica e durante todos os procedimentos realizados no estudo.

\section{Intervenção - protocolo de treinamento}

Primeiramente, os sinais vitais (frequência cardíaca e pressão arterial) das participantes foram verificados. Se estivessem dentro dos padrões normais, iniciava-se o treinamento.

As participantes foram submetidas ao protocolo de caminhada, com duração de 30 minutos, média de tempo considerada necessária para ocorrência de benefícios [4,5], durante quatro semanas, totalizando 10 sessões $[15,16]$. Antes da intervenção as participantes realizaram aquecimento na esteira ergométrica e, após a intervenção, um protocolo de desaceleração, ambos por cinco minutos a $2,5 \mathrm{~km} / \mathrm{h}$.

O exercício aeróbico foi realizado em esteira elétrica ergométrica (Gii Movement modelo LX160, Barueri, SP), sem inclinação e com a intensidade determinada pela frequência cardíaca, calculada pela fórmula de Karvonnen, utilizando em torno de $60 \%$ da frequência cardíaca máxima [6]. Durante todas as sessões a frequência cardíaca foi monitorada continuamente por meio de um frequencímetro (Nonin Onyx 9500, Plymouth, MN, EUA) a fim de garantir a intensidade proposta, assim como a integridade da participante. Ao final da sessão, novamente os sinais vitais foram avaliados.

\section{Análise estatística}

As medidas antropométricas entre grupos foram comparadas por meio do Teste $\mathrm{t}$ independente. Para verificar a esfericidade, a homogeneidade e a distribuição normal dos dados foram aplicados os testes de Levene, Mauchly e Shapiro-Wilk, respectivamente. As comparações intragrupos e intergrupos foram realizados por meio da análise de variância (ANOVA) com delineamento misto. A correção de Bonferroni foi utilizada como post hoc. Como forma de explorar mais os dados e verificar a existência de diferenças entre os grupos foi realizada a comparação entre as variações percentuais. As análises estatísticas foram realizadas no software SPSS Statistics 17 versão 17. Foi adotado o nível de significância de $5 \%(p=0,05)$.

\section{RESULTADOS}

No total, 31 mulheres apresentaram-se como voluntárias. Destas, oito não foram incluídas por não atenderem ao critério de nível de atividade física e três foram excluídas por terem sofrido lesões musculoesqueléticas nos últimos seis meses, permanecendo um total de 20 participantes, sendo 10 alocadas no GE e 10 no GC.

As comparações realizadas pelo teste $t$ independente mostraram idade, massa e altura similares entre os grupos, o que exclui a influência dessas características nos resultados do estudo (Tabela 1). Não foram encontradas diferenças significativas para o GC quanto às medidas referentes à avaliação inicial e final para nenhuma das variáveis de interesse $(\mathrm{F}=1,01 ; \mathrm{p}=0,943)$ (Tabela 2). Da mesma forma, o GE não apresentou diferença significativa quanto às medidas referentes à avaliação pré e pós-intervenção $(\mathrm{F}=2,71 ; \mathrm{p}=0,201)$ (Tabela 3$)$.

Tabela 1. Dados antropométricos das 20 mulheres saudáveis com idade entre 18 e 30 anos, participantes do estudo.

\begin{tabular}{|lcc|}
\hline Antropometria & $\begin{array}{c}\text { Grupo Controle } \\
\mathbf{N}=\mathbf{1 0} \\
\text { Média } \pm(\mathrm{DP})\end{array}$ & $\begin{array}{c}\text { Grupo Exercício } \\
\mathbf{N}=\mathbf{1 0} \\
\text { Média } \pm(\mathrm{DP})\end{array}$ \\
\hline Idade (anos) & $22,0 \pm 3,6$ & $21,9 \pm 2,7$ \\
Peso $(\mathrm{Kg})$ & $62,3 \pm 7,3$ & $65,7 \pm 10,7$ \\
Altura $(\mathrm{m})$ & $1,65 \pm 0,04$ & $1,65 \pm 0,05$ \\
\hline
\end{tabular}

Teste $\mathrm{T}$ independente.

Tabela 2. Grupo Controle - Acuidade proprioceptiva do tornozelo, medida por meio do instrumento Star Excursion Balance Test. Médias referentes às avaliações inicial e final do grupo controle, constituído por 10 mulheres adultas saudáveis, orientadas a manter suas atividades normais entre as avaliações.

\begin{tabular}{|c|c|c|c|c|c|c|c|c|}
\hline & \multicolumn{8}{|c|}{ Linhas de direção } \\
\hline & M1 & M2 & M3 & M4 & M5 & L1 & L2 & L3 \\
\hline Avaliação Inicial & 60,00 & 58,70 & 59,00 & 60,30 & 61,70 & 57,00 & 54,20 & 51,40 \\
\hline Avaliação Final & 60,00 & 60,20 & 60,80 & 56,70 & 54,80 & 52,70 & 53,00 & 52,20 \\
\hline $\mathbf{p}^{*}$ & 0,10 & 0,41 & 0,44 & 0,36 & 0,99 & 0,68 & 0,76 & 0,27 \\
\hline
\end{tabular}

M1, distância entre o ponto central e a linha anterior; M2, distância entre o ponto central e a linha ântero-medial; M3, distância entre o ponto central e a linha medial; M4, distância entre o ponto central e a linha póstero-medial; M5, distância entre o ponto central e a linha posterior; L1, distância entre o ponto central e a linha anterolateral; L2, distância entre o ponto central e a linha lateral; L3, distância entre o ponto central e a linha póstero-lateral. Valores das variáveis expressos em centímetros.

* Análise de Variância com delineamento misto. 
Tabela 3. Grupo Exercício - Acuidade proprioceptiva do tornozelo, medida por meio do instrumento Star Excursion Balance Test. Médias referentes às avaliações inicial e final do grupo de intervenção, constituído por 10 mulheres adultas saudáveis, submetidas a um programa de exercícios físicos entre as duas avaliações.

\begin{tabular}{lccccccccc}
\hline & \multicolumn{9}{c}{ Linhas de direção } \\
\cline { 2 - 9 } & M1 & M2 & M3 & M4 & M5 & L1 & L2 & L3 \\
\hline Avaliação inicial (pré-intervenção) & 68,60 & 68,50 & 70,30 & 70,50 & 67,40 & 65,80 & 58,00 & 58,50 \\
Avaliação final (pós-intervenção) & 69,90 & 69,90 & 68,70 & 65,60 & 67,70 & 59,50 & 60,10 & 58,70 \\
\hline p* & 0,61 & 0,34 & 0,42 & 0,85 & 0,78 & 0,12 & 0,09 & 0,18 \\
\hline
\end{tabular}

M1, distância entre o ponto central e a linha anterior; M2, distância entre o ponto central e a linha ântero-medial; M3, distância entre o ponto central e a linha medial; M4, distância entre o ponto central e a linha póstero-medial; M5, distância entre o ponto central e a linha posterior; L1, distância entre o ponto central e a linha anterolateral; L2, distância entre o ponto central e a linha lateral; L3, distância entre o ponto central e a linha póstero-lateral. Valores das variáveis expressos em centímetros.

* Análise de Variância com delineamento misto.

Tabela 4. Variação percentual entre as médias da acuidade proprioceptiva do tornozelo, medida por meio do instrumento Star Excursion Balance Test, entre as avaliações inicial e final do Grupo Controle e do Grupo Exercício, em 20 mulheres adultas saudáveis, sendo o Grupo Exercício submetido a um programa de exercícios físicos entre as duas avaliações.

\begin{tabular}{|c|c|c|c|c|c|c|c|c|c|}
\hline & \multicolumn{8}{|c|}{ Linhas de direção } & \multirow{2}{*}{ p-valor } \\
\hline & M1 & M2 & M3 & M4 & M5 & L1 & L2 & L3 & \\
\hline Controle & 0 & 2,55 & 3,05 & $-5,97$ & $-11,18$ & $-7,54$ & $-2,21$ & 1,55 & \multirow{2}{*}{0,675} \\
\hline Exercício & 1,89 & 2,04 & $-2,27$ & $-6,95$ & 0,44 & $-9,57$ & 3,62 & 0,34 & \\
\hline
\end{tabular}

M1, distância entre o ponto central e a linha anterior; M2, distância entre o ponto central e a linha ântero-medial; M3, distância entre o ponto central e a linha medial; M4, distância entre o ponto central e a linha póstero-medial; M5, distância entre o ponto central e a linha posterior; L1, distância entre o ponto central e a linha anterolateral; L2, distância entre o ponto central e a linha lateral; L3, distância entre o ponto central e a linha póstero-lateral. Valores das variáveis expressos em centímetros.

Em relação à comparação entre grupos, não houve variações significativas tanto no momento pré-exercício $(\mathrm{F}=1,27, \mathrm{p}=0,452)$ quanto no momento pós-exercício $(\mathrm{F}=1,31, \mathrm{p}=0,164)$ (Tabela 4$)$.

\section{DISCUSSÃO}

Os presentes achados sugerem que um programa de caminha em esteira composto por 10 sessões com duração de 30 minutos não proporcionou mudanças na acuidade proprioceptiva do tornozelo em mulheres jovens saudáveis. $\mathrm{O}$ fato de não ocorrerem mudanças proprioceptivas após a caminha em esteira pode ser justificado, entre outros fatores, pela hipótese de que essa atividade não promove ativação satisfatória dos estabilizadores dinâmicos. O recrutamento desses músculos ocorre em situações que exigem maior grau de equilíbrio, ou seja, em situações que necessitem de correção do posicionamento do centro de massa, como atividades em terrenos irregulares, com olhos vendados e em apoio unipodal [8]. Portanto, parece que o caráter pouco desafiador da atividade proposta, no âmbito proprioceptivo e muscular, pode ter tido influência na falta de ganho proprioceptivo que ocorreu na amostra estudada.

Além disso, parece que o principal fator responsável por ganhos proprioceptivos é a multidirecionali- dade [9]. Estudos vêm mostrando que a adição de exercícios em diversas direções promovem ganhos expressivos na acuidade proprioceptiva [8]. A atividade realizada neste estudo era unidirecional, o que pode ter contribuído para a falta do efeito em estudo. Um estudo que avaliou a acuidade proprioceptiva após a corrida também não encontrou resultados significativos [7], o que sugere que exercícios unidirecionais não estimulem adaptações do sistema proprioceptivo. A corrida é uma atividade que necessita maior aporte muscular para a manutenção do equilíbrio, no entanto mesmo assim não foram observados resultados positivos em relação à acuidade proprioceptiva [7]. Portanto, parece que a realização de atividades multidirecionais é mais impactante em termos proprioceptivos do que a realização de atividades que promovem a ativação dos estabilizadores dinâmicos.

Os benefícios da atividade física na acuidade proprioceptiva foram observados em um estudo que comparou jovens saudáveis com idosas que praticavam atividade física regularmente, não havendo diferença significativa entre os grupos [10]. Esses resultados sugerem que a prática regular de exercícios pode atenuar os déficits proprioceptivos adquiridos com a idade. Dessa forma, parece que a atividade física regular não é capaz de promover adaptações proprioceptivas em adultos jovens, entretanto, ela tem a 
capacidade de prevenir déficits proprioceptivos relativos ao envelhecimento.

Algumas limitações deste estudo devem ser abordadas. O reduzido tamanho amostral pode ter diminuído o poder final do estudo. Em segundo lugar, a inclusão apenas de mulheres jovens saudáveis e sem histórico de lesões talvez não tenha permitido observar se o programa de caminhada em esteira poderia ter caráter preventivo em indivíduos com alguma lesão prévia. É possível que indivíduos com acuidade proprioceptiva alterada se beneficiassem de um programa de caminhada em esteira. Em terceiro, apenas uma tentativa do teste proprioceptivo foi mensurada; talvez, o mais indicado seria fazer mais tentativas e calcular a média dos escores. Por fim, não foram observadas quantas tentativas cada participante necessitou para realizar a tarefa antes e após a intervenção, o que poderia trazer mais informações sobre a influência do procedimento experimental. Considerando tais limitações, seria interessante a realização de estudos futuros que investiguem o efeito da caminhada em mulheres com lesões prévias de tornozelo e, também, se mulheres em diferentes faixas etárias apresentariam diferentes respostas ao protocolo, uma vez que se sabe que com o avançar da idade o sistema proprioceptivo fica menos efetivo [4].

Considerando os presentes resultados, conclui-se que um programa de 10 sessões de caminhada em esteira não foi capaz de promover adaptações na acuidade proprioceptiva do tornozelo em mulheres jovens saudáveis, nas condições testadas.

\section{REFERÊNCIAS}

1. Carvalho AR de. Utilização do treinamento neuromuscular e proprioceptivo para prevenção das lesões desportivas. Arq Ciênc Saúde UNIPAR. 2010;14:269-76.

2. Shields RK, Madhavan S, Cole KR, Brostad JD, DeMeulenaere JL, Eggers CD, et al. Proprioceptive coordination of movement sequences in humans. Clin Neurophysiol. 2005;116:87-92. http://dx.doi.org/10.1016/j.clinph.2004.07.019

3. Ergen E, Ulkar B. Proprioception and Ankle Injuries in Soccer. Clin Sports Med. 2008;27:195-217. http://dx.doi.org/10.1016/j.csm.2007.10.002

4. Zazulak BT, Hewett TE, Reeves NP, Goldberg B, Cholewicki J. The Effects of Core Proprioception on Knee Injury: A Prospective BiomechanicalEpidemiological Study. Am J Sports Med. 2006;35:368-73. http://dx.doi.org/10.1177/0363546506297909

5. Han J, Anson J, Waddington G, Adams R, Liu Y. The Role of Ankle Proprioception for Balance Control in relation to Sports Performance and Injury. Biomed Res Int. 2015;2015. http://dx.doi.org/10.1155/2015/842804

6. Gribble P a., Hertel J, Plisky P. Using the star excursion balance test to assess dynamic postural-control deficits and outcomes in lower extremity injury: A literature and systematic review. J Athl Train. 2012;47(3):339-57. http://dx.doi.org/10.4085/1062-6050-47.3.08

7. Stergioulas A, Trippolitsioti A, Kostopoulos N, Gavriilidis A, Sotiropoulos M BP. Amateur basketball injuries. A prospective study among male and female athletes. Biol Excercise. 2007;3:36-42.

8. Ness BM, Taylor AL, Haberl MD. Clinical Observation and Analysis of Movement Quality During Performance on the Star Exersion Balance Test. Int J Sports Phys Ther. 2015;10:168-77.

9. Hughes T, Rochester P. The effects of proprioceptive exercise and taping on proprioception in subjects with functional ankle instability: A review of the literature. Phys Ther Sport. 2008;9:136-47. http://dx.doi.org/10.1016/j.ptsp.2008.06.003

10. Lima MMO, Britto RR, Baião EA, Alves GS, Abreu CDG, Parreira VF. Exercício aeróbico no controle da hipertensão arterial na pós-menopausa. Fisiot Mov. 2011;24:23-31.

11. Hanson S, Jones A. Is there evidence that walking groups have health benefits? A systematic review and meta-analysis. Br J Sports Med. 2015;49:710-5. http://dx.doi.org/10.1136/bjsports-2014-094157

12. Imai A, Kaneoka K, Okubo Y, Shiraki H. Comparison of the immediate effect of different types of trunk exercise on the star excursion balance test in male adolescent soccer players. Int J Sports Phys Ther. 2014;9:428-35.

13. Craig CL, Marshall AL, Sjöström M, Bauman AE, Booth ML, Ainsworth BE, Pratt M, Ekelund U, Yngve A, Sallis JF, Oja P. International physical activity questionnaire: 12-Country reliability and validity. Med Sci Sports Exerc. 2003;35:1381-95. http://dx.doi.org/10.1249/01. MSS.0000078924.61453.FB

14. Hallal PC, Gomez LF, Parra DC, Lobelo F, Mosquera J, Florindo AA, Reis RS, Pratt M, Sarmiento OL. Lessons learned after 10 years of IPAQ use in Brazil and Colombia. J Phys Act Health. 2010;7 Suppl 2:S259-64.

15. Hess DM, Joyce CJ, Arnold BL, Gansneder BM. Effect of a 4-week agility-training program on postural sway in the functionally unstable ankle. J Sport Rehabil. 2001;10:24-35.

16. Rozzi SL, Lephart SM, Sterner R, Kuligowski L. Balance training for persons with functionally unstable ankles. J Orthop Sports Phys Ther. 1999;29:478-86. http://dx.doi.org/10.2519/jospt.1999.29.8.478 C 